\title{
Prognostic and Predictive Value of the Pretreatment Albumin-bilirubin Score for Short and Long-term Survival in Patients With Non-Hodgkin Lymphoma- associated Hemophagocytic Lymphohistiocytosis With Hepatic Injuries
}

\section{Guangli Yin}

Jiangsu Province Hospital and Nanjing Medical University First Affiliated Hospital

\section{Wanying Cheng}

Jiangsu Province Hospital and Nanjing Medical University First Affiliated Hospital

\section{Yongqian Shu}

Jiangsu Province Hospital and Nanjing Medical University First Affiliated Hospital

Hongxia Qiu ( $\nabla$ qhx9805@126.com )

Jiangsu Province Hospital and Nanjing Medical University First Affiliated Hospital

https://orcid.org/0000-0002-6348-4888

\section{Limin Duan}

Jiangsu Province Hospital and Nanjing Medical University First Affiliated Hospital

\section{Research}

Keywords: hemophagocytic lymphohistiocytosis, non-Hodgkin lymphoma, ALBI score, ferritin, hepatic injuries, mortality

Posted Date: January 4th, 2022

DOI: https://doi.org/10.21203/rs.3.rs-1151055/v1

License: (c) (1) This work is licensed under a Creative Commons Attribution 4.0 International License.

Read Full License 


\section{Abstract}

Purpose: This study aimed to assess the prognostic value of pretreatment albumin-bilirubin (ALBI) on short-term mortality (30 days) and long-term ( $\geq 1$ year) survival

The aim of this study was to construct a prognosis model of non-Hodgkin lymphoma-associated secondary hemophagocytic lymphohistiocytosis (NHL-sHLH) patients with hepatic injuries by the combination of ALBI score and clinical parameters.

Material and methods: This retrospective study included $168 \mathrm{NHL}$-sHLH patients with hepatic injuries between February 1, 2014, and February 1, 2020. Multivariable logistic/Cox models and restricted cubic spline models were conducted to evaluate the relationships between the ALBI score and short- and longterm survival. The predictive performance of the ALBI score was assessed and compared using timedependent receiver operating characteristic (ROC) analysis.

Results: Among 168 adult NHL-sHLH patients, 82 (48.8\%) patients died within 30 days after admission, and 144 (85.7\%) patients died during the follow-up period. Multivariate logistic regression indicated that ALBI grade could be an independent risk factor for predicting the prognosis of patients with 30-day mortality and overall survival (odds ratios $[\mathrm{OR}]_{30}$ days $5.37,95 \%$ confidence interval 2.41-12.64, $P<0.001$; hazard ratios $[\mathrm{HR}]_{\mathrm{OS}} 1.52,95 \%$ confidence interval $\left.1.06-2.18, P=0.023\right)$, respectively. The restricted cubic spline curve displayed a linear and positive relationship between the ALBI score and risk of mortality ( $P$ for nonlinearity $=0.503)$. Furthermore, receiver operating characteristic $(R O C)$ curve analysis showed that the area under the curve (AUC) for predicting mortality by integrative analysis of the ALBI score and ferritin was significantly improved compared to the ALBI score (AUC 30 days: 0.820 vs $0.693, P=0.001$; $\mathrm{AUC}_{1 \text { year: }}: 0.754$ vs $\left.0.681, P=0.043\right)$ or ferritin $\left(\mathrm{AUC}_{30 \text { days }}: 0.820\right.$ vs $0.724, P=0.005 ; \mathrm{AUC}_{1 \text { year: }}: 0.754$ vs $0.658, P=0.031)$ alone.

Conclusions: These results suggest that the ALBI score could be a useful indicator of 30-day mortality and overall survival ( $\geq 1$ year) for NHL-sHLH patients with hepatic injuries, and the combination analysis of the ALBI score and ferritin provides incremental prognostic value for clinical use.

\section{Introduction}

Secondary hemophagocytic lymphohistiocytosis (sHLH) is a rare life-threatening systemic disease characterized by the uncontrolled activation of T cells and macrophages, producing overwhelming hypercytokinemia and leading to multiple organ failure ${ }^{1}$. sHLH is often associated with neoplastic diseases, including lymphoma, viral infections, and autoimmune or autoinflammatory disorders ${ }^{2}$. Of these, sHLH in the context of lymphoma is considered a major challenge to clinicians due to variable overlaps of symptoms, such as hepatic dysfunction and multiorgan failure, thus resulting in a higher incidence of mortality ${ }^{3}$. Although serum ferritin has been validated to predict outcomes ${ }^{4,5}$, it is not refined enough to evaluate prognosis in NHL-sHLH patients with hepatic injuries. Hepatic injuries, which present 
with a wide range of hepatic dysfunction, are recognized as one of the major complications in patients with sHLH and sometimes cause acute liver failure (ALF), which can contribute to high syndromic mortality ${ }^{6,7}$. In a previous report of $12 \mathrm{HLH}$ patient autopsies, $75 \%(9 / 12)$ of patients had evidence of hepatocellular injury, and all autopsies revealed significant liver injury, including necrosis, fibrosis, and cholestasis ${ }^{8}$.

However, sHLH at presentation is usually accompanied by coagulopathy, and liver biopsy cannot be routinely obtained to assess liver injury ${ }^{9}$. It is therefore necessary to develop a tool to assess the severity of hepatic dysfunction. Recently, the albumin-bilirubin (ALBI) score/grade was proposed to predict liver dysfunction and survival status among patients with hepatocellular carcinoma but was also validated in patients with liver cirrhosis without nonhepatocellular carcinoma, acute heart failure, and acute pancreatiti ${ }^{10-13}$. Moreover, the levels of serum albumin and total bilirubin can previously reflect liver function and prognosis in sHLH patients ${ }^{14}$.

Using this background, we conducted the present study to evaluate the ALBI score/grade and potential predictive risk of patients with NHL-sHLH with hepatic injuries.

\section{Patients And Methods}

\section{Study Patients}

The clinical and laboratory data were extracted from the hospital database of 168 consecutive NHL-sHLH patients with hepatic injuries at our center from February 1, 2014, to February 1, 2019, for this retrospective study. All the enrolled patients for whom the new pathological diagnosis of NHL met WHO pathological criteria for biopsy samples ${ }^{15}$ or patients for whom the diagnosis of NHL was based on MICM (morphology, flow cytometric immunophenotype, IgH or TCR rearrangement and immunohistochemistry) of bone marrow biopsy criteria ${ }^{16}$. Meanwhile, NHL patients were assessed to confirm HLH diagnosis by the HLH-2004 diagnostic criteria of the Histiocyte Society ${ }^{17}$ and HScore ${ }^{18}$ before treatment. The exclusion criteria were as follows: 1) patients who were diagnosed with nonlymphoma-associated sHLH ( $n=87)$ and Hodgkin lymphoma-associated $\mathrm{sHLH}(n=4) ; 2)$ patients who had no hepatic injuries (normal serum aminotransaminase, albumin, bilirubin levels, and no hepatomegaly) $(n=22) ; 3)$ patients who had a history of cirrhosis or other serious liver diseases with decompensated liver function as well as ascites and jaundice; 4) patients who had missing data on albumin and bilirubin variables ( $n=5)$; and 5) patients who had no follow-up data $(n=3)$. Our study was approved by the Ethics Committee of the First Affiliated Hospital of Nanjing Medical University (Clinical Trial: ChiCTR2000032421), in accordance with the guidelines of the 1975 Declaration of Helsinki. Informed consent was obtained to review patient medical records.

\section{Calculation of ALBI score and definition of hepatic injury}


The ALBI score was calculated before treatment using appropriate clinical parameters and recommended methods ${ }^{19}$. The ALBI score was calculated by the formula: $\left(0.66 \times \log _{10}\right.$ bilirubin $)-(0.085 \times$ albumin $)$. ALBI grades were defined as ALBI grade 1 (score $\leq-2.60$ ), ALBI grade 2 (score $>-2.60$ and $\leq-1.39$ ) and ALBI grade 3 (score > -1.39). During calculation, serum bilirubin was expressed in $\mu \mathrm{mol} / \mathrm{L}$, and serum albumin level was expressed in $\mathrm{g} / \mathrm{L}$. According to previous literature reports, hepatic injury/dysfunction was defined as at least two of the following: 1) elevated bilirubin, 2) elevated alanine and aspartate aminotransferase (ALT, AST) levels, 3) low albumin levels, 4) coagulopathy and 5) hepatomegaly9, 20.

\section{Covariate collection and follow-up}

Patients' clinical characteristics assessed at the first admission included age; sex; fever; complete blood cell counts; hepatosplenomegaly; and blood biochemical tests (including albumin, bilirubin, aspartate aminotransferase (AST), alanine aminotransferase (ALT), triglycerides (TG), lactate dehydrogenase $(L D H)$ ), fibrinogen (FIB), ferritin, and serum soluble interleukin-2 receptor (sIL-2R, sCD25) were reviewed from their medical records on admission. Epstein-Barr virus (EBV) was evaluated by both serology and EBV DNA real-time quantitative polymerase chain reaction (RQ-PCR) analysis. The NK-cell cytotoxicity assay is not available at our facility. Bone marrow aspiration and biopsy samples were reviewed at the first diagnosis. The primary outcome of the current study was overall survival (OS), which was calculated as the time in days from NHL-sHLH first diagnosis to the date of death from any cause or the last followup. The secondary outcome was 30-day mortality, which described the survival status in the first 30 days on admission.

\section{Treatments}

After the diagnosis was confirmed, patients were reviewed at our multidisciplinary HLH team for treatment recommendations. Among these 168 patients, 124 patients $(71.9 \%)$ had received various kinds of chemotherapy, including 62 patients treated with a CEOP \pm R-based regimen (cyclophosphamide, vincristine, etoposide, prednisone, and/or rituximab), 23 treated with the SMILE regimen (dexamethasone, methotrexate, ifosfamide, L-asparaginase, and etoposide), 18 treated with the DEP regimen (liposomal doxorubicin, etoposide, and methylprednisolone), 14 treated with the LMED regimen (methotrexate, etoposide, L-asparaginase, and dexamethasone), 4 treated with the P-GemOx regimen (pegaspargase, gemcitabine, oxaliplatin), 2 treated with anti-programmed death 1 antibody, and 1 treated with a modified hypercytbine-CVAD regimen (cyclophosphamide, doxorubicin, vincristine and dexamethasone alternating with rituximab, high-dose methotrexate and cytarabine). A median of 1 cycle (range 1-6 cycles) was given. The other 44 patients received only the HLH-94 protocol or steroid and/or etoposide and intravenous immunoglobulin therapies.

\section{Statistical analysis}

Continuous variables are presented as the mean \pm standard derivation or median (with interquartile range). Category variables are expressed as frequencies or percentages. The comparison of survival probability was performed by the Kaplan-Meier method with the log-rank test. Univariate logistic regression and Cox proportional hazards models were used to estimate odds ratios (ORs)/hazard ratios (HRs) and 95\% 
confidence intervals (95\% Cls) for associations between clinical and laboratory prognostic factors and 30-day mortality as well as overall survival outcomes. Multivariable regressions with stepwise forward selection were performed to analyze the influence of relevant variables (variables with $P<0.05$ in univariate Cox regression were subsequently entered into the model) on survival outcomes. We log (10)transformed ferritin and SCD25 variables because they were skewed. Restricted cubic splines with three knots placed at the 10th, 50th and 90th percentiles (the number of knots was selected according to the Akaike information criterion) were generated to examine them after adjusting for confounding factors to examine whether nonlinear relationships existed between the ALBI score and the risk of mortality, and the tests for nonlinearity were calculated by Wald $\chi 2$ tests. The predictive powers of the ALBI score, serum ferritin, and their combination were evaluated using receiver operator characteristic (ROC) curve analysis.

All statistical analyses were performed with $\mathrm{R}$ software version 3.6.0 (R Foundation for Statistical Computing, Vienna, Austria) and STATA/MP statistical software (version 14.0; StataCorp, TX, USA), and two-sided $P$ values $<0.05$ were considered statistically significant.

\section{Results}

\section{Baseline characteristics}

According to the protocol (Figure 1), 168 patients were ultimately included. Detailed demographic and clinical features of the NHL-sHLH patients with hepatic injuries are summarized in Table 1 . The majority of patients were male (70.8\%). The median age was 54 years (range $41-64$ years) at the time of sHLH diagnosis. According to the sHLH etiologies ${ }^{1}$, T cell or NK/T cell lymphoma $(106,63.1 \%)$ was the most frequent underlying disease. Stratification by ALBI grade identified 83 patients with ALBI grade $2(49.4 \%)$, 83 patients with ALBI grade $3(49.4 \%)$, and only 2 patients with ALBI grade $1(1.2 \%)$. Owing to a small number of patients with ALBI grade 1, they were combined with the patients with ALBI grade 2 . 
Table 1

Baseline characteristics of NHL-sHLH patients with hepatic injuries

\begin{tabular}{|c|c|}
\hline Characteristics & Value $(N=168)$ \\
\hline Age (years) & $54(41-64)$ \\
\hline $\operatorname{Sex}(M: F)$ & 119: 49 \\
\hline ANC, $\times 10^{9} / \mathrm{L}$ & $1.27(0.62-2.00)$ \\
\hline$<1.0$ & $68(40.5)$ \\
\hline$\geq 1.0$ & $100(59.5)$ \\
\hline$H B, g / L$ & $81.50(69.00-96.00)$ \\
\hline$<90$ & $106(63.1)$ \\
\hline$\geq 90$ & $62(36.9)$ \\
\hline $\mathrm{PLT}, \times 10^{9} / \mathrm{L}$ & $39.00(20.00-69.75)$ \\
\hline$<100$ & $149(88.7)$ \\
\hline$\geq 100$ & $19(11.3)$ \\
\hline ALB, $g / L$ & $27.22 \pm 4.94$ \\
\hline TBIL, umol/L & $19.25(13.10-39.70)$ \\
\hline $\mathrm{ALT}, \mathrm{U} / \mathrm{L}$ & $54.25(32.80-111.85)$ \\
\hline AST, U/L & $86.85(44.63-198.18)$ \\
\hline $\mathrm{LDH}, \mathrm{U} / \mathrm{L}$ & $740.00(412.50-1404.25)$ \\
\hline $\mathrm{TG}, \mathrm{mmol} / \mathrm{L}$ & $2.46(1.74-3.73)$ \\
\hline$<3.0$ & $102(60.7)$ \\
\hline$\geq 3.0$ & $66(39.3)$ \\
\hline $\mathrm{FIB}, \mathrm{g} / \mathrm{L}$ & $1.44(0.99-2.20)$ \\
\hline$\leq 1.5$ & $90(53.6)$ \\
\hline$>1.5$ & $78(46.4)$ \\
\hline Ferritin, ug/L & $4290.50(1506.00-13927.50)$ \\
\hline sCD25, ng/L & $40587.00(22768.00-52839.00)$ \\
\hline Splenomegaly, n (\%) & $157(93.5)$ \\
\hline Hepatomegaly, n (\%) & $63(37.5)$ \\
\hline
\end{tabular}


Hemophagocytic, n (\%)

HScore, points

EBV infection, $n(\%)$

Type of lymphoma, n (\%)

B-sHLH

T/NKT-sHLH

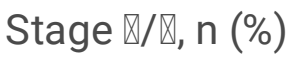

B symptoms, n (\%)

IPI score

ALBI grade, $n(\%)$
145 (86.3)

237 (203-274)

$92(54.8)$
62 (36.9)

$106(63.1)$

151(89.9)

$160(95.2)$

$3.3 \pm 0.7$
1

2

3
$2(1.2)$

83 (49.4)

83 (49.4)

Abbreviations: ANC, absolute neutrophil count; HB, hemoglobin; PLT, platelet; ALB, albumin; TBIL, Total bilirubin; ALT, alanine transaminase; AST, aspartate transaminase; LDH, lactate dehydrogenase; TG, triglyceride; FIB, fibrinogen; sCD25, soluble interleukin-2 receptor; EBV, Epstein-Barr virus; B-sHLH, B cell lymphoma associated haemophagocytic lymphohistiocytosis; T/NKT-sHLH, T cell lymphoma or NK/T cell lymphoma associated haemophagocytic lymphohistiocytosis; ALBI, albumin- bilirubin; PALBI, platelet- albumin- bilirubin.

Data are means \pm standard deviation or medians, with interquartile range in parentheses.

\section{Association between ALBI and study outcomes}

The median OS was 36 days (95\% Cl 15-175 days), 144 patients died, and 24 survived through the entire follow-up period. Factors that were found to be significantly associated with 30-day mortality and overall survival are listed in Table 2 and Table 3 according to univariate analysis $(P<0.05)$. After adjusting for variables including laboratory examinations and traditional HLH risk factors, ALBI grade 3 was identified as an independent predictor of 30 -day mortality $(\mathrm{OR}=5.37,95 \% \mathrm{Cl}: 2.41-12.64, P<0.001)$ and overall survival ( $\mathrm{HR}=1.52,95 \% \mathrm{Cl}: 1.06-2.18, P=0.023)$, and ferritin was also independently associated with an increased risk of 30 -day mortality $(\mathrm{OR}=4.13,95 \% \mathrm{Cl}$ : $1.73-10.42, P=0.002)$ and overall survival $(\mathrm{HR}=$ $1.68,95 \% \mathrm{Cl}: 1.12-2.53, P=0.012)$. The Kaplan-Meier survival analysis showed that the median OS was 90.0 days for patients with ALBI grade $1 / 2$ and 21.0 days for ALBI grade $3(P<0.0001)$ (Figure 2). When the ALBI score was analyzed as a continuous variable and controlling for the same variables, the estimated risk associated with mortality increased incrementally at gradually increasing scores ( $P$ for nonlinearity $=0.503$, Figure 3 ). 
Table 2

Univariate and multivariate analysis of predictors for 30 days mortality.

\begin{tabular}{|c|c|c|c|c|}
\hline \multirow[t]{2}{*}{ Variables (Ref) } & \multicolumn{2}{|l|}{ Univariate analyses } & \multicolumn{2}{|c|}{ Multivariate analyses } \\
\hline & $\mathrm{HR}(95 \% \mathrm{Cl})$ & $P$ & $\mathrm{HR}(95 \% \mathrm{Cl})$ & $P$ \\
\hline Male (female) & $0.63(0.32-1.24)$ & 0.185 & & \\
\hline Age ( $\leq 60$ years) & $0.97(0.52-1.82)$ & 0.933 & & \\
\hline ANC $\left(\geq 1.0 \times 10^{9} / \mathrm{L}\right)$ & $1.32(0.71-2.45)$ & 0.377 & & \\
\hline $\mathrm{HB}(\geq 90 \mathrm{~g} / \mathrm{L})$ & $0.58(0.31-1.10)$ & 0.094 & & \\
\hline $\operatorname{PLT}\left(\geq 100 \times 10^{9} / \mathrm{L}\right)$ & $6.02(1.68-21.53)$ & 0.006 & & \\
\hline $\mathrm{FIB}(>1.5 \mathrm{~g} / \mathrm{L})$ & $1.80(0.97-3.32)$ & 0.061 & & \\
\hline TG (<3.0mmol/L) & $1.79(0.96-3.35)$ & 0.069 & & \\
\hline $\mathrm{LDH}(\leq 2.5 \times \mathrm{ULN})$ & $1.35(0.73-2.48)$ & 0.338 & & \\
\hline $\operatorname{ALT}(\leq 2.5 \times \mathrm{ULN})$ & $1.32(0.63-2.79)$ & 0.467 & & \\
\hline $\mathrm{AST}(\leq 2.5 \times \mathrm{ULN})$ & $2.51(1.35-4.69)$ & 0.004 & & \\
\hline Splenomegaly & $1.19(0.78-2.01)$ & 0.078 & & \\
\hline Hepatomegaly & $2.39(1.14-5.00)$ & 0.021 & & \\
\hline Hemophagocytosis & $0.86(0.36-2.06)$ & 0.728 & & \\
\hline $\log _{10}$ Ferritin ug/L & $5.07(2.55-10.10)$ & $<0.001$ & $4.13(1.73-10.42)$ & 0.002 \\
\hline $\log _{10} \mathrm{sCD} 25 \mathrm{ng} / \mathrm{L}$ & $2.46(0.97-6.25)$ & 0.059 & & \\
\hline EBV (no infection) & $1.11(0.61-2.04)$ & 0.734 & & \\
\hline T/NKT-sHLH (B-sHLH) & $2.65(1.38-5.08)$ & 0.003 & & \\
\hline HLH04/Chemotherapy (GC \pm VP16 \pm IVlg) & $0.13(0.06-0.31)$ & $<0.001$ & $0.23(0.08,0.59)$ & 0.004 \\
\hline ALB, $g / L$ & $0.07(0.01-0.40)$ & 0.003 & & \\
\hline TBIL, umom/L & $2.23(1.50-3.32)$ & $<0.001$ & & \\
\hline \multicolumn{5}{|c|}{$\begin{array}{l}\text { Abbreviations: ANC, absolute neutrophil count; HB, hemoglobin; PLT, platelet; ALB, albumin; TBIL, } \\
\text { Total bilirubin; ALT, alanine transaminase; AST, aspartate transaminase; LDH, lactate dehydrogenase; } \\
\text { TG, triglyceride; FIB, fibrinogen; SCD25, soluble interleukin-2 receptor; EBV, Epstein-Barr virus; B-sHLH, } \\
\text { B cell lymphoma associated haemophagocytic lymphohistiocytosis; T/NKT-sHLH, T cell lymphoma or } \\
\text { NK/T cell lymphoma associated haemophagocytic lymphohistiocytosis; GC, glucocorticoid; IVlg, } \\
\text { intravenous immunoglobulins; VP16, etoposide; ALBI, albumin- bilirubin. }\end{array}$} \\
\hline \multicolumn{5}{|c|}{ OR, odds ratio; $95 \% \mathrm{Cl}, 95 \%$ confidence interval } \\
\hline Bold statistical significance & & & & \\
\hline
\end{tabular}




\begin{tabular}{|c|c|c|c|c|}
\hline \multirow[t]{2}{*}{ Variables (Ref) } & \multicolumn{2}{|c|}{ Univariate analyses } & \multicolumn{2}{|c|}{ Multivariate analyses } \\
\hline & $\mathrm{HR}(95 \% \mathrm{Cl})$ & $P$ & $\mathrm{HR}(95 \% \mathrm{Cl})$ & $P$ \\
\hline \multicolumn{5}{|l|}{ ALBI } \\
\hline grade 3 (grade $1+2$ ) & $5.90(3.07-11.67)$ & $<0.001$ & $5.37(2.41-12.64)$ & $<0.001$ \\
\hline \multicolumn{5}{|c|}{$\begin{array}{l}\text { Abbreviations: ANC, absolute neutrophil count; HB, hemoglobin; PLT, platelet; ALB, albumin; TBIL, } \\
\text { Total bilirubin; ALT, alanine transaminase; AST, aspartate transaminase; LDH, lactate dehydrogenase; } \\
\text { TG, triglyceride; FIB, fibrinogen; SCD25, soluble interleukin-2 receptor; EBV, Epstein-Barr virus; B-sHLH, } \\
\text { B cell lymphoma associated haemophagocytic lymphohistiocytosis; T/NKT-sHLH, T cell lymphoma or } \\
\text { NK/T cell lymphoma associated haemophagocytic lymphohistiocytosis; GC, glucocorticoid; IVIg, } \\
\text { intravenous immunoglobulins; VP16, etoposide; ALBI, albumin- bilirubin. }\end{array}$} \\
\hline \multicolumn{5}{|c|}{ OR, odds ratio; $95 \% \mathrm{Cl}, 95 \%$ confidence interval } \\
\hline Bold statistical significance & & & & \\
\hline
\end{tabular}


Table 3

Univariate and multivariate analysis of predictors for overall survival.

\begin{tabular}{|c|c|c|c|c|}
\hline \multirow[t]{2}{*}{ Variables (Ref) } & \multicolumn{2}{|c|}{ Univariate analyses } & \multicolumn{2}{|c|}{ Multivariate analyses } \\
\hline & $\mathrm{HR}(95 \% \mathrm{Cl})$ & $P$ & $\mathrm{HR}(95 \% \mathrm{Cl})$ & $P$ \\
\hline Male (female) & $1.15(0.80-1.66)$ & 0.441 & & \\
\hline Age ( $\leq 60$ years) & $1.01(0.72-1.42)$ & 0.956 & & \\
\hline ANC $(\geq 1.0 \times 109 / L)$ & $1.20(0.86-1.67)$ & 0.289 & & \\
\hline $\mathrm{HB}(\geq 90 \mathrm{~g} / \mathrm{L})$ & $1.25(0.89-1.77)$ & 0.200 & & \\
\hline PLT ( $\geq 100 \times 109 / L)$ & $2.25(1.24-4.07)$ & 0.007 & $1.94(1.03-3.63)$ & 0.040 \\
\hline $\mathrm{FIB}(>1.5 \mathrm{~g} / \mathrm{L})$ & $1.61(1.15-2.25)$ & 0.005 & & \\
\hline $\mathrm{TG}(<3.0 \mathrm{mmol} / \mathrm{L})$ & $1.46(1.04-2.03)$ & 0.027 & & \\
\hline $\operatorname{LDH}(\leq 2.5 \times \mathrm{ULN})$ & $1.26(0.91-1.76)$ & 0.164 & & \\
\hline $\operatorname{ALT}(\leq 2.5 \times \cup L N)$ & $1.08(1.72-0.63)$ & 0.697 & & \\
\hline $\operatorname{AST}(\leq 2.5 \times \cup L N)$ & $1.55(1.11-2.16)$ & 0.009 & & \\
\hline Splenomegaly & $1.47(0.87-2.31)$ & 0.069 & & \\
\hline Hepatomegaly & $1.46(1.00-2.14)$ & 0.049 & & \\
\hline Hemophagocytosis & $1.17(0.72-1.90)$ & 0.522 & & \\
\hline Log10Ferritin ug/L & $2.32(1.63-3.32)$ & $<0.001$ & $1.68(1.12-2.53)$ & 0.012 \\
\hline Log10sCD25 ng/L & $1.69(0.99-2.88)$ & 0.051 & & \\
\hline EBV (no infection) & $1.36(0.97-1.89)$ & 0.074 & & \\
\hline T/NKT-sHLH (B-sHLH) & $1.86(1.31-2.65)$ & $<0.001$ & $1.53(1.03-2.25)$ & 0.033 \\
\hline HLH04/Chemotherapy (GC \pm VP16 \pm IVlg) & $0.31(0.21-0.45)$ & $<0.001$ & $0.36(0.24,0.53)$ & $<0.001$ \\
\hline ALB g/L & $0.29(0.12-0.70)$ & 0.006 & & \\
\hline TBIL umom/L & $1.58(1.32-1.89)$ & $<0.001$ & & \\
\hline
\end{tabular}

Abbreviations: ANC, absolute neutrophil count; HB, hemoglobin; PLT, platelet; ALB, albumin; TBIL, Total bilirubin; ALT, alanine transaminase; AST, aspartate transaminase; $L D H$, lactate dehydrogenase; TG, triglyceride; FIB, fibrinogen; SCD25, soluble interleukin-2 receptor; EBV, Epstein-Barr virus; B-sHLH, B cell lymphoma associated haemophagocytic lymphohistiocytosis; T/NKT-sHLH, T cell lymphoma or NK/T cell lymphoma associated haemophagocytic lymphohistiocytosis; GC, glucocorticoid; IVlg, intravenous immunoglobulins; VP16, etoposide; ALBI, albumin- bilirubin.

OR, odds ratio; $\mathrm{HR}$, hazards ratio; $95 \% \mathrm{Cl}, 95 \%$ confidence interval

Bold statistical significance 


\begin{tabular}{|llll|}
\hline Variables (Ref) & \multicolumn{2}{l}{ Univariate analyses } & \multicolumn{2}{l|}{ Multivariate analyses } \\
\cline { 2 - 4 } & HR $(95 \%$ Cl) & $P$ & HR $(95 \%$ Cl)
\end{tabular}

\section{The ALBI score improves risk prediction according to time- dependent ROC analysis.}

Figure 4 shows the AUCs for the ALBI score and serum ferritin levels at 30 days and 1 year after the start of follow-up using time-dependent ROC analysis. Ferritin combined with the ALBI score showed the highest accuracy in predicting 30-day and 1-year mortality compared to either the ferritin $\left(\mathrm{AUC}_{30}\right.$ days: 0.820 vs $0.724, P=0.005$ AUC $_{1 \text { year }} 0.754$ vs $0.658, P=0.031$ ) or ALBI score (AUC 30 days: 0.820 vs 0.693 , $P=0.001 ; \mathrm{AUC}_{1 \text { year: }}: 0.754$ vs $0.681, P=0.043$ ) alone.

\section{Discussion}

In this retrospective study, the ALBI score was associated with an increased risk of mortality among NHLsHLH patients with hepatic injuries. The combination of pretreatment ALBI score and serum ferritin, a benchmark prognostic marker in sHLH patients, improved prognostic prediction discrimination. Our data revealed that ALBI was a better prognostic tool in predicting short-term (30 days) or long-term ( $\geq 1$ year) survival, which persisted after extensive adjustment of confounding variables, including demographic, clinical, laboratory examinations and traditional sHLH risk factors. Moreover, we demonstrated that the ALBI score has a direct positive association with mortality in the total cohort by restricted cubic spline (RCS) modeling. In addition, the combination of the ALBI score and ferritin provided incremental prognostic value for each measure separately for both 30-day and 1-year survival. These results suggest that the ALBI score is a better predictive marker for survival in patients with hepatic injuries.

Lymphoma is a common trigger identified in adult $\mathrm{SHLH}$, especially non-Hodgkin lymphoma-associated sHLH (NHL-sHLH), which has a high mortality rate, with an estimated 30-day survival of approximately 
$56-70 \%$ and 2-year survival of $8-34.3 \%{ }^{21-23}$. These patients frequently present a combination of persistent fever, elevated aminotransferase (AST, ALT), decreased serum albumin, jaundice, and hepatomegaly or splenomegaly. Several studies have evaluated whether sHLH patients with hepatic involvement have a poorer prognosis 8,14 ; therefore, early prediction of survival outcome contributes greatly to patient management and aids in making appropriate risk assessments as well as treatment decisions. Clinically, the ALBI score has been widely used for assessing liver function and predicting survival outcomes in various diseases with liver dysfunction. Takeshi et al prospectively evaluated the ALBI grade as a predictor of survival in a multicenter cohort of 1,190 patients with acute heart failure (AHF). After adjusting for pre-existing prognostic factors, the ALBI score was associated with 1-year mortality $(\mathrm{HR}=2.11,95 \% \mathrm{Cl}: 1.60-2.79, P<0.001)^{12}$. Zhang also showed similar findings; the ALBI grade appears to be a promising prognostic biomarker associated with overall survival (HR=1.60, 95\% Cl: $1.12-$ 2.29, $P=0.02$ ) in critically ill patients with acute pancreatitis ${ }^{13}$. Moreover, several studies have described that serum albumin and total bilirubin are significantly associated with mortality in $\mathrm{HLH}$ patients ${ }^{8,24}$. Our study, by multivariate regression analysis and RCS modeling, clearly demonstrated that ALBI was strongly associated with survival outcomes (both 30-day mortality and overall survival ( $\geq 1$ year)). In addition, our investigation in this study demonstrated that the combination analysis of ALBI score and ferritin had a significant improvement over either ALBI score (AUC 30 days: 0.820 vs $0.693, P=0.001 ; A_{1} C_{1}$ year: 0.754 vs $0.681, P=0.043)$ or ferritin $\left(\right.$ AUC $_{30}$ days: 0.820 vs $0.724, P=0.005$; AUC $_{1 \text { year }}: 0.754$ vs 0.658 , $P=0.031)$ alone.

The increased risk of mortality associated with a higher ALBI score might be ascribed to uncontrolled proliferation of cytotoxic CD8+ T lymphocytes (CTLs) and macrophages, creating an uncontrolled loop of inflammation that is responsible for hepatic injuries. ALBI, consisting of serum albumin and total bilirubin, could reflect the systemic inflammatory response. On the one hand, serum albumin plays an important role in modulating systemic inflammatory reactions and organic oxidation resistance ${ }^{25}$; on the other hand, serum total bilirubin is always elevated with hepatobiliary dysfunction in response to various cytokines in circulating blood, such as interferon-g (IFN- $\gamma$ ), interleukin-1beta (IL-1 $\beta$ ), and interleukin-6 (IL$6)^{8}$. All of the above mechanisms, alone or together, may be attributed to decreased serum albumin and elevated total bilirubin and indirectly reflect the degree of hepatic injury.

Our findings provide references that routine clinical laboratory assays such as serum albumin and total bilirubin can be used to identify patients at higher risk of death while applying an advanced evaluation for NHL-sHLH patients. This is the first study that addressed the ALBI score, a promising hepatic injury tool, and the risk of mortality (both short-term and long-term survival). The ALBI score improved risk predictions of mortality in both the short and long term and could provide clinical guidance for timely multidisciplinary discussions of when to initiate HLH-directed immune suppressants and NHL-directed intensive chemotherapy. However, several limitations of our study were noted. First, the enrolled study population was small, and the study was retrospective in nature. Second, although we had fully adjusted a broad set of covariates, we could not rule out the role of unmeasured or unrecognized confounders. 
Finally, the observational study could only demonstrate the association between ALBI score/grade and the prognosis of patients but could not provide conclusions for causality.

\section{Conclusion}

Our study demonstrated that the ALBI score was associated with 30-day mortality and long-term ( $\geq 1$ year) survival among NHL-sHLH patients with hepatic injuries. Early detection based on a higher ALBI score/risk grade could be helpful in ensuring prompt interventions to reduce mortality.

\section{Abbreviations}

NHL-sHLH, non-Hodgkin lymphoma-associated secondary hemophagocytic lymphohistiocytosis; ALBI, albumin-bilirubin; ANC, absolute neutrophil count; ALF, acute liver failure; MICM, Morphology Immunology, Cytogenetics, Molecular biology; HB, hemoglobin; PLT, platelet; ALT, alanine transaminase; AST, aspartate transaminase; $L D H$, lactate dehydrogenase; TG, triglyceride; FIB, fibrinogen; SCD25, soluble interleukin-2 receptor; EBV, Epstein-Barr-virus; RQ-PCR, real-time quantitative polymerase chain reaction; BsHLH, B cell lymphoma associated secondary hemophagocytic lymphohistiocytosis; T/NKT-sHLH, T cell lymphoma or NK/T cell lymphoma associated secondary hemophagocytic lymphohistiocytosis; GC, glucocorticoid; IVIg, intravenous immunoglobulins; VP16, etoposide; ROC, receiver operating characteristic; OR, odds ratio; HR, hazards ratio; $95 \% \mathrm{Cl}, 95 \%$ confidence interval

\section{Declarations}

\section{Acknowledgements}

Authors expressed their gratitude for all persons who were involved in reporting patients and gathering data.

\section{Consent for publication}

Consent for publication was obtained from all participants.

\section{Availability of data and material}

The data of our patients is available in the department of medical records at Jiangsu Province Hospital and the First Affiliated Hospital of Nanjing Medical University. This data can be released with the agreement of the patients and is available from the corresponding author upon reasonable request.

\section{Author contributions}

GLY and HXQ designed the experiment. GLY performed the experiments. GLY, WYC, YQS, LMD and HXQ organized the clinical materials. GLY and WYC performed the data analysis and wrote the paper. All authors contributed to the final approval of the manuscript. 


\section{Funding}

This study was supported by the National Natural Science Foundation of China (grant number 81570175).

\section{Ethics approval and consent to participate}

Our study was approved by the ethics committee of the First Affiliated Hospital of Nanjing Medical University and registered on the Chinese Clinical Trial Registry (ChiCTR2000032421).

\section{Competing interests}

The authors declare that they have no competing interests.

\section{References}

1. Al-Samkari H, Berliner N: Hemophagocytic Lymphohistiocytosis. Annual review of pathology 2018, 13:27-49.

2. Hayden A, Park S, Giustini D, Lee AY, Chen LY: Hemophagocytic syndromes (HPSs) including hemophagocytic lymphohistiocytosis $(\mathrm{HLH})$ in adults: A systematic scoping review. Blood reviews 2016, 30:411-20.

3. Li F, Yang Y, Jin F, Dehoedt C, Rao J, Zhou Y, Li P, Yang G, Wang M, Zhang R, Yang Y: Clinical characteristics and prognostic factors of adult hemophagocytic syndrome patients: a retrospective study of increasing awareness of a disease from a single-center in China. Orphanet journal of rare diseases 2015, 10:20.

4. Zhou J, Zhou J, Wu ZQ, Goyal H, Xu HG: Ferritin index is a strong prognostic marker in adult hemophagocytic lymphohistiocytosis. International journal of clinical practice 2020:e13704.

5. Otrock ZK, Eby CS: Clinical characteristics, prognostic factors, and outcomes of adult patients with hemophagocytic lymphohistiocytosis. American journal of hematology 2015, 90:220-4.

6. Jordan MB, Allen CE, Weitzman S, Filipovich AH, McClain KL: How I treat hemophagocytic lymphohistiocytosis. Blood 2011, 118:4041-52.

7. Cappell MS, Hader I, Amin M: Acute liver failure secondary to severe systemic disease from fatal hemophagocytic lymphohistiocytosis: Case report and systematic literature review. World journal of hepatology 2018, 10:629-36.

8. Goldman J, Desai MS, McClain KL, Tcharmtchi MH, Kennedy CE, Thompson K, Lam F, Bashir DA, Chinn IK, Goldberg BR, Allen CE, Nguyen TC: Hepatobiliary Dysfunction and Disseminated Intravascular Coagulation Increase Risk of Mortality in Pediatric Hemophagocytic Lymphohistiocytosis. Pediatric critical care medicine : a journal of the Society of Critical Care Medicine and the World Federation of Pediatric Intensive and Critical Care Societies 2018, 19:e522e30. 
9. Guthery SL, Heubi JE: Liver involvement in childhood histiocytic syndromes. Current opinion in gastroenterology 2001, 17:474-8.

10. Hansmann J, Evers MJ, Bui JT, Lokken RP, Lipnik AJ, Gaba RC, Ray CE, Jr.: Albumin-Bilirubin and Platelet-Albumin-Bilirubin Grades Accurately Predict Overall Survival in High-Risk Patients Undergoing Conventional Transarterial Chemoembolization for Hepatocellular Carcinoma. Journal of vascular and interventional radiology : JVIR 2017, 28:1224-31 e2.

11. Lu LH, Zhang YF, Mu-Yan C, Kan A, Zhong XP, Mei J, Ling YH, Li SH, Shi M, Wei W, Guo RP: Plateletalbumin-bilirubin grade: Risk stratification of liver failure, prognosis after resection for hepatocellular carcinoma. Digestive and liver disease : official journal of the Italian Society of Gastroenterology and the Italian Association for the Study of the Liver 2019, 51:1430-7.

12. Matsue Y, Kagiyama N, Yamaguchi T, Kuroda S, Okumura T, Kida K, Mizuno A, Oishi S, Inuzuka Y, Akiyama E, Matsukawa R, Kato K, Suzuki S, Naruke T, Yoshioka K, Miyoshi T, Baba Y, Yamamoto M, Mizutani K, Yoshida K, Kitai T: Clinical and Prognostic Values of ALBI Score in Patients With Acute Heart Failure. Heart, lung \& circulation 2020, 29:1328-37.

13. Shi L, Zhang D, Zhang J: Albumin-bilirubin score is associated with in-hospital mortality in critically ill patients with acute pancreatitis. European journal of gastroenterology \& hepatology 2020, 32:96370.

14. Arca M, Fardet L, Galicier L, Riviere S, Marzac C, Aumont C, Lambotte O, Coppo P: Prognostic factors of early death in a cohort of 162 adult haemophagocytic syndrome: impact of triggering disease and early treatment with etoposide. British journal of haematology 2015, 168:63-8.

15. Cazzola M: Introduction to a review series: the 2016 revision of the WHO classification of tumors of hematopoietic and lymphoid tissues. Blood 2016, 127:2361-4.

16. Liang JH, Wang L, Zhu HY, Qian J, Liao H, Wu JZ, Xia Y, Wu W, Cao L, Fan L, Li JY, Xu W: Doseadjusted EPOCH regimen as first-line treatment for non-Hodgkin lymphoma-associated hemophagocytic lymphohistiocytosis: a single-arm, open-label, phase II trial. Haematologica 2020, 105:e29-e32.

17. Henter JI, Horne A, Arico M, Egeler RM, Filipovich AH, Imashuku S, Ladisch S, McClain K, Webb D, Winiarski J, Janka G: HLH-2004: Diagnostic and therapeutic guidelines for hemophagocytic lymphohistiocytosis. Pediatric blood \& cancer 2007, 48:124-31.

18. Fardet L, Galicier L, Lambotte O, Marzac C, Aumont C, Chahwan D, Coppo P, Hejblum G: Development and validation of the HScore, a score for the diagnosis of reactive hemophagocytic syndrome. Arthritis \& rheumatology 2014, 66:2613-20.

19. Liu PH, Hsu CY, Hsia CY, Lee YH, Chiou YY, Huang YH, Lee FY, Lin HC, Hou MC, Huo TI: ALBI and PALBI grade predict survival for HCC across treatment modalities and BCLC stages in the MELD Era. Journal of gastroenterology and hepatology 2017, 32:879-86.

20. Beken B, Aytac S, Balta G, Kuskonmaz B, Uckan D, Unal S, Cetin M, Gumruk F: The clinical and laboratory evaluation of familial hemophagocytic lymphohistiocytosis and the importance of hepatic and spinal cord involvement: a single center experience. Haematologica 2018, 103:231-6. 
21. Lehmberg K, Nichols KE, Henter JI, Girschikofsky M, Greenwood T, Jordan M, Kumar A, Minkov M, La Rosee P, Weitzman S, Study Group on Hemophagocytic Lymphohistiocytosis Subtypes of the Histiocyte S: Consensus recommendations for the diagnosis and management of hemophagocytic lymphohistiocytosis associated with malignancies. Haematologica 2015, 100:997-1004.

22. Sano H, Kobayashi R, Tanaka J, Hashino S, Ota S, Torimoto Y, Kakinoki Y, Yamamoto S, Kurosawa M, Hatakeyama N, Haseyama Y, Sakai H, Sato K, Fukuhara T: Risk factor analysis of non-Hodgkin lymphoma-associated haemophagocytic syndromes: a multicentre study. British journal of haematology 2014, 165:786-92.

23. Chang Y, Cui M, Fu X, Han L, Zhang L, Li L, Li X, Sun Z, Wu J, Zhang X, Li Z, Nan F, Yan J, Sheng G, Zhang M: Lymphoma associated hemophagocytic syndrome: A single-center retrospective study. Oncology letters 2018, 16:1275-84.

24. Malinowska I, Machaczka M, Popko K, Siwicka A, Salamonowicz M, Nasilowska-Adamska B: Hemophagocytic syndrome in children and adults. Archivum immunologiae et therapiae experimentalis 2014, 62:385-94.

25. Arroyo V, Garcia-Martinez R, Salvatella X: Human serum albumin, systemic inflammation, and cirrhosis. Journal of hepatology 2014, 61:396-407.

\section{Figures}


A total of 289 consecutive sHLH patients were admitted to First Affiliated Hospital of Nanjing Medical University between February 1, 2014, and February 1, 2020

$\checkmark$\begin{tabular}{l}
$\begin{array}{l}\cdot \text { Patients were diagnosed non-lymphoma associated sHLH } \\
(\mathrm{n}=87) \\
\text { Patients were diagnosed Hodgkin lymphoma associated } \\
\mathrm{sHLH}(\mathrm{n}=4)\end{array}$ \\
\hline
\end{tabular}

Non-Hodgkin lymphoma associated sHLH $(\mathrm{n}=198)$

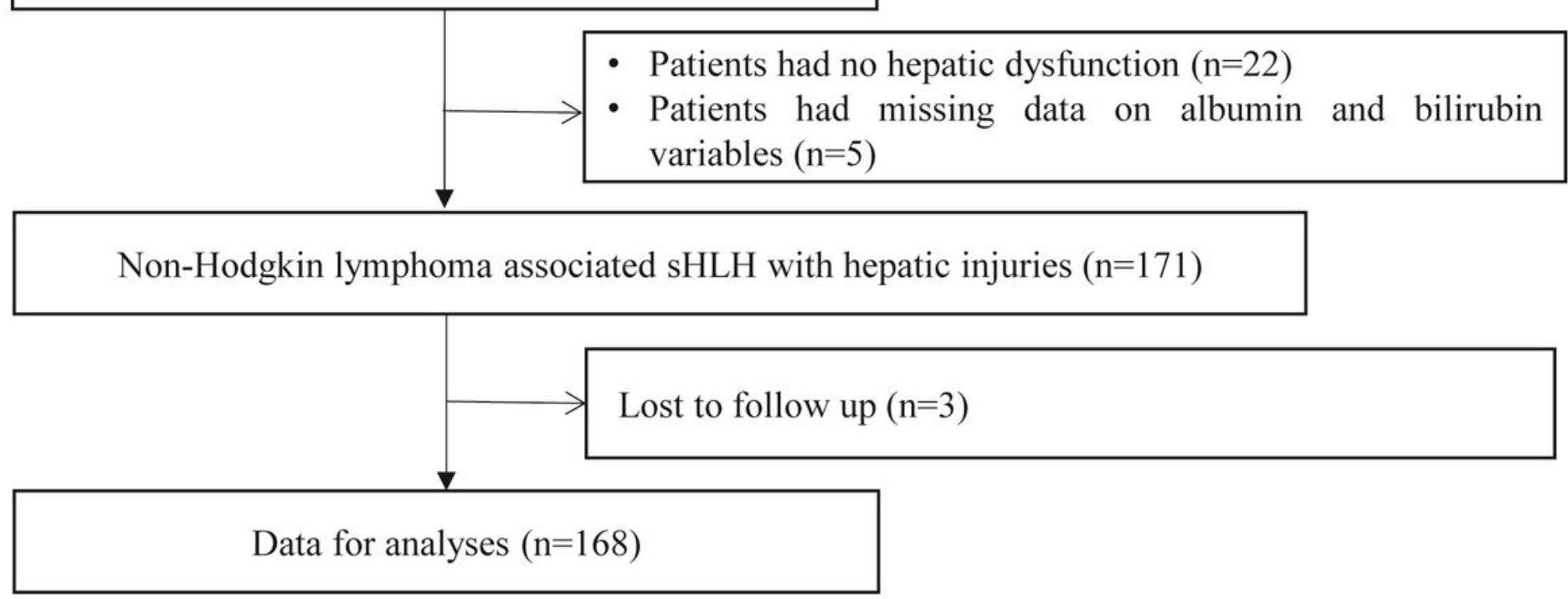

\section{Figure 1}

Flow diagram of the study population. SHLH, secondary hemophagocytic lymphohistiocytosis. 


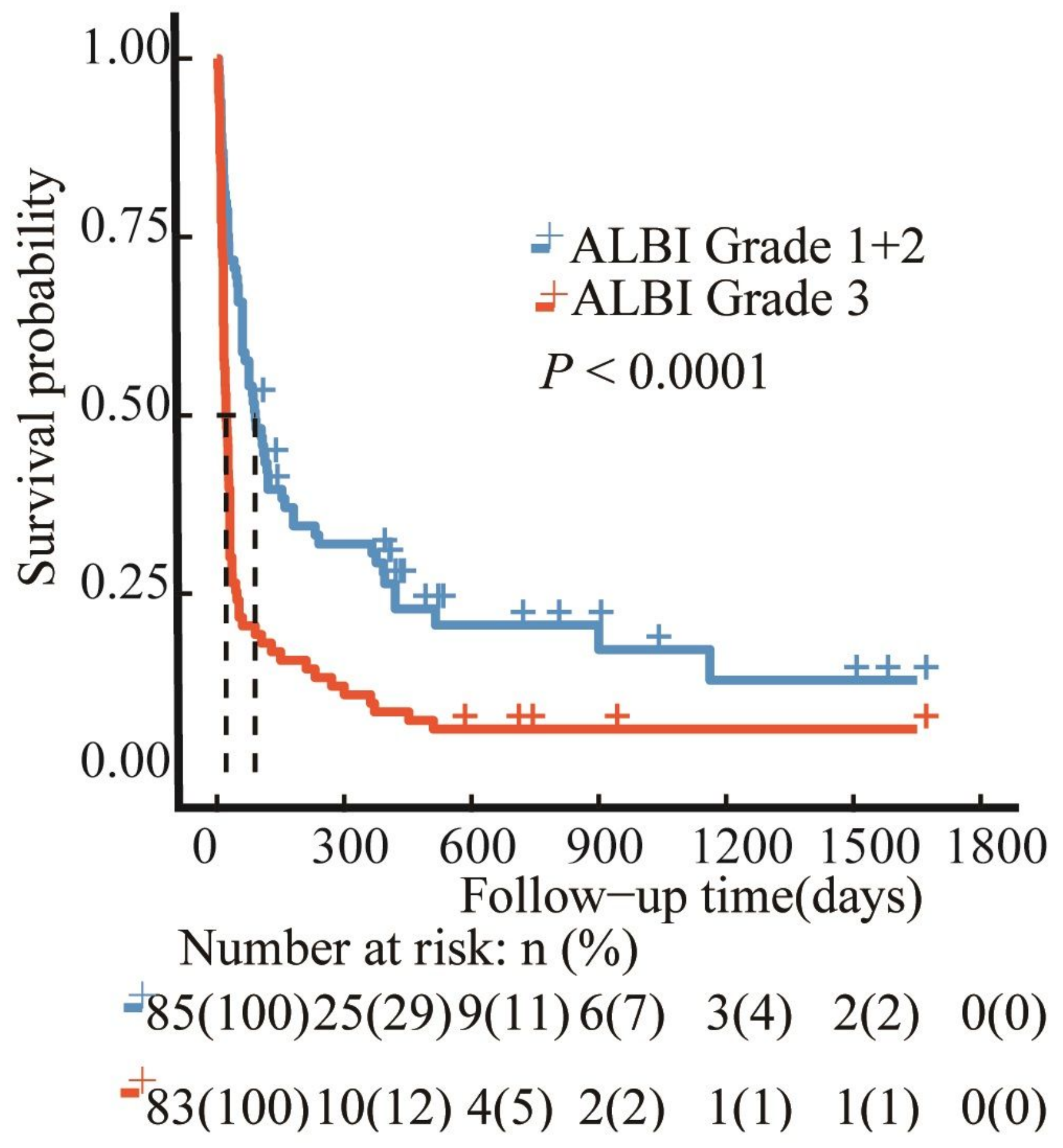

Figure 2

Survival curves of the NHL-sHLH patients according to ALBI grade. ALBI, albumin-bilirubin. 
A

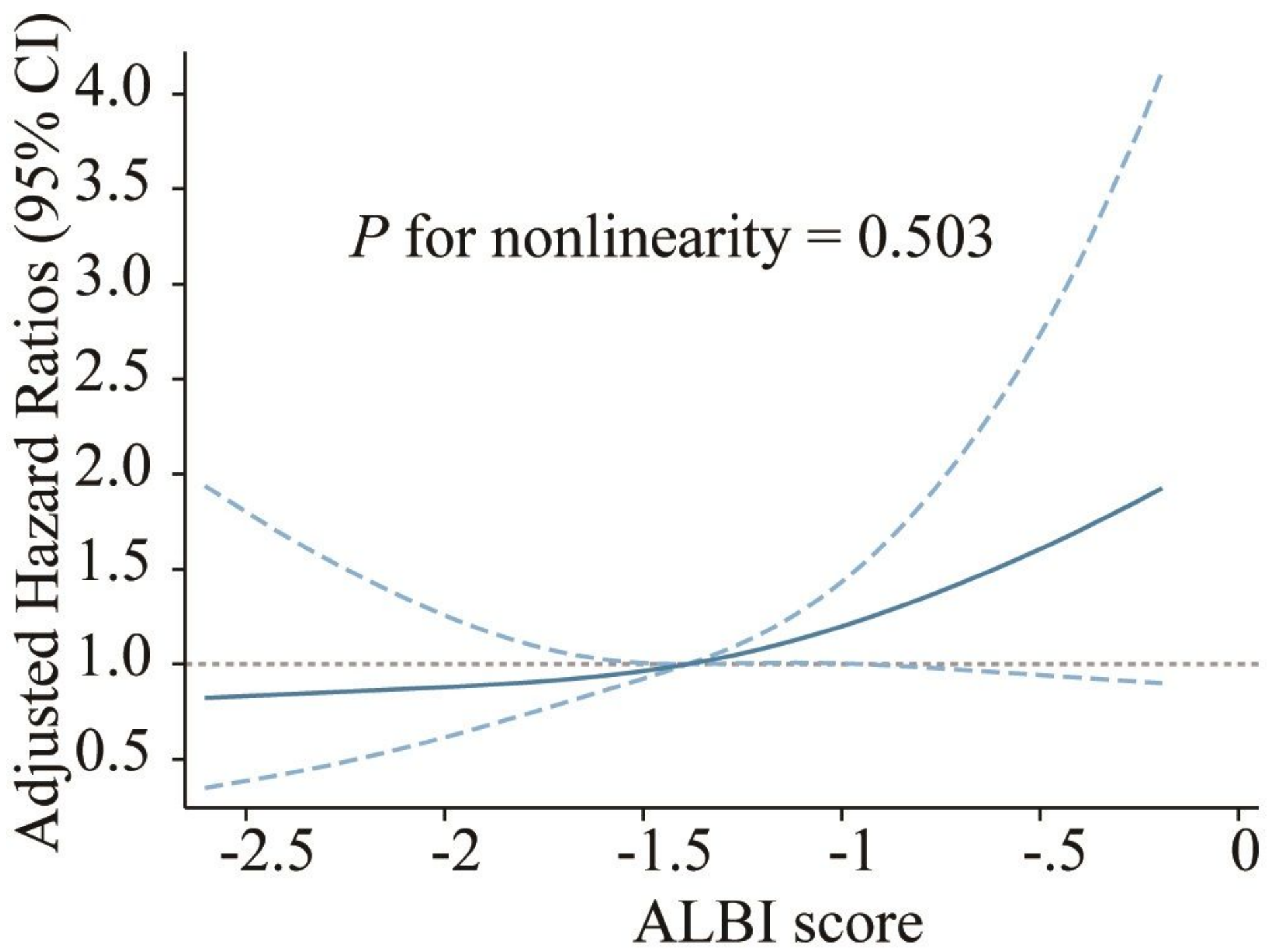

Figure 3

Cubic spline plot of the association between the ALBI score and risk of mortality among adult NHL-sHLH patients. The solid line and dashed line represent the estimated hazard ratios and their corresponding $95 \%$ Cls, respectively. Analyses were adjusted for PLT, AST, FIB, TG, hepatomegaly, ferritin, etiologies, and treatment strategies. ALBI, albumin-bilirubin; NHL, non-Hodgkin lymphoma; $\mathrm{HLH}$, hemophagocytic lymphohistiocytosis; PLT, platelet; AST, aspartate transaminase; FIB, fibrinogen; TG, triglyceride. 
A

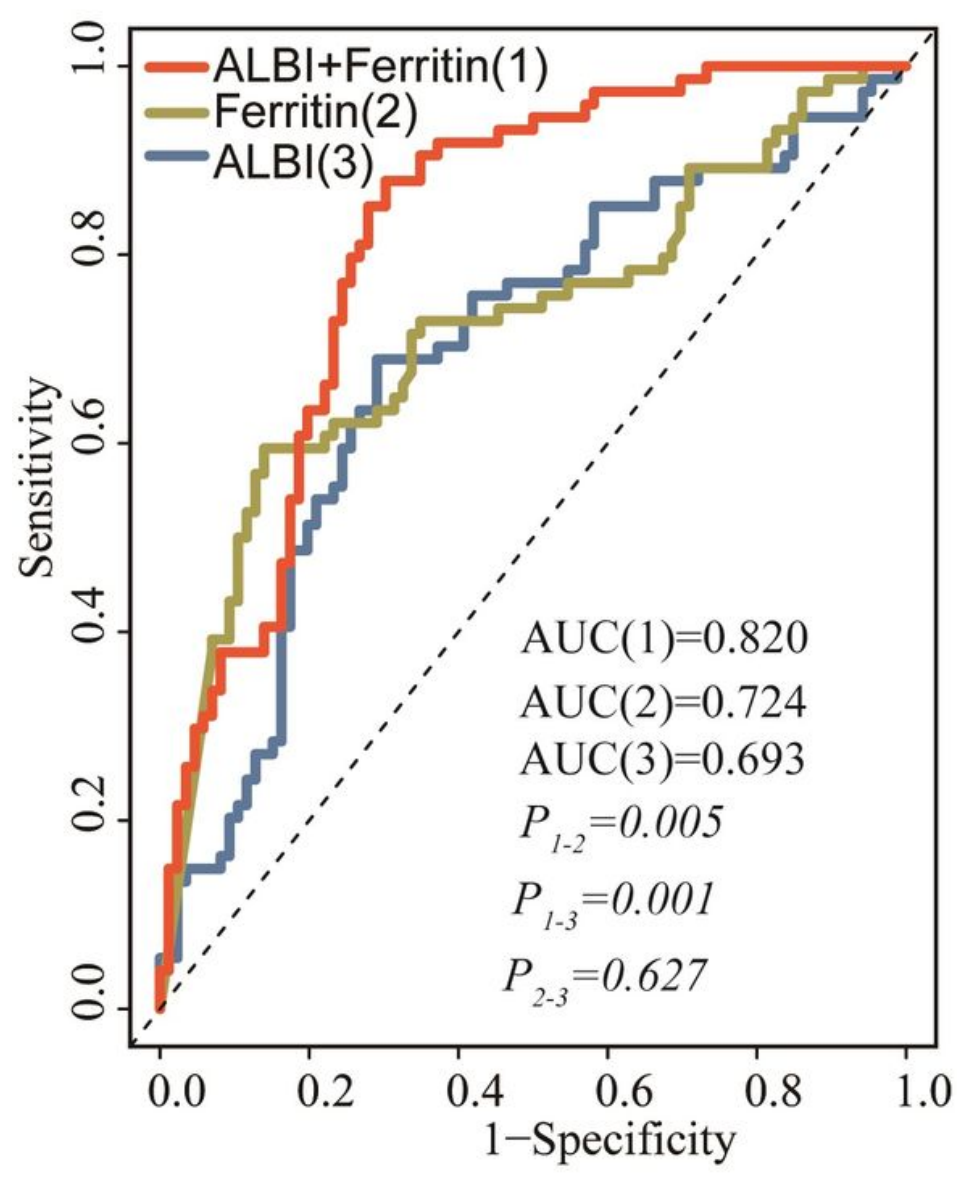

$\mathrm{B}$

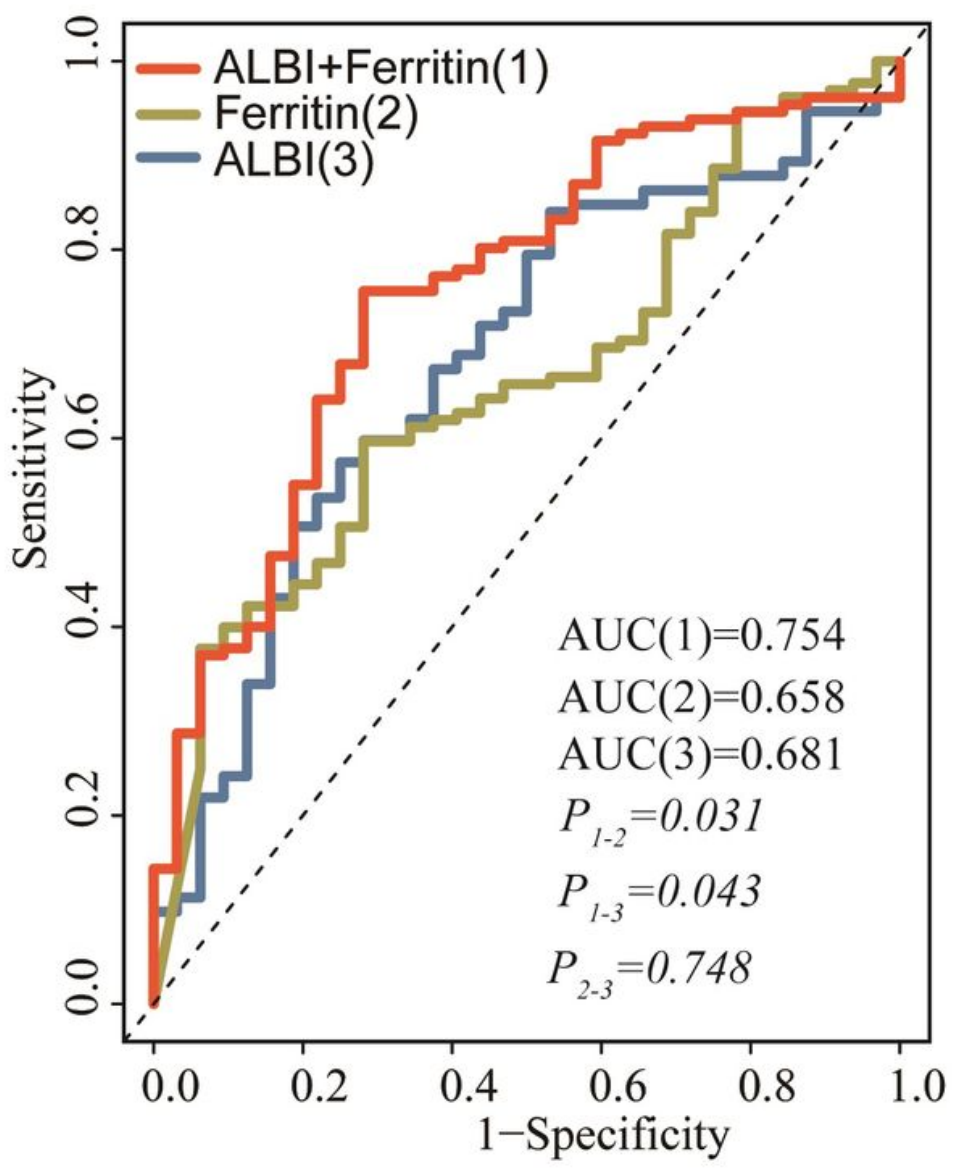

Figure 4

Time-dependent ROC analysis for the ALBI score, ferritin and their combination in predicting survival at 30 days $(A)$ and after 1 year (B) of follow-up. ALBI, albumin-bilirubin. 